\title{
An Application of Fractional Brownian Motion to Vessel Identification Based on its Sound Spectrum
}

\author{
M. Wiciak ${ }^{a, *}$, I. GLOZA ${ }^{b}$, R. JÓŹWIAK ${ }^{b}$ AND P. WiciaK ${ }^{c}$ \\ ${ }^{a}$ Cracow University of Technology, Institute of Mathematics, Warszawska 24, 31-155 Krakow, Poland \\ ${ }^{b}$ Polish Naval Academy, Śmigowicza 69, 81-103 Gdynia, Poland \\ ${ }^{c}$ AGH University of Science and Technology, Mickiewicza 30, 30-059 Krakow, Poland
}

\begin{abstract}
The paper deals with the problem of vessel identification. The presented method is based on fractional Brownian analysis of vessel power spectrum. The measurements for three vessels were carried out with the use of a mobile measuring module in the Gulf of Gdansk; next, the information obtained from sound spectra was identified. Two classifiers connected with fractional Brownian motion were used: the first-order increments and the standard deviation. Finally, classification decision was made using the Mahalanobis distance. Numerical experiments were performed using MATLAB.
\end{abstract}

DOI: 10.12693 /APhysPolA.128.A-46

PACS: 43.60.Lq

\section{Introduction}

Problem of ship identification by its radiated sound is regarded as difficult. This is by the reason of its complexity [1-2]. On the other hand, vessel recognition is very important, particularly from the military point of view. In the literature, different methods are presented, from traditional line spectrum and average spectrum analysis, through wavelet analysis, Bayesian analysis, and neural networks, to fractal analysis [3-6]. Among a number of studies, those concerned with detection of objects buried in the sea bottom or source localization in water are important and challenging [7-11]. The aim of this paper is to present a method of vessel identification based on fractional Brownian analysis of its power spectrum. In [3], the Authors suggest that ship sound can be treated as fractal signals. This is thanks to [12-14] where it has been shown that the power spectra of ship sound decrease by 6 decibels per octave at frequencies, and are similar to $1 / f$ signals. Using this hypothesis, the classification process for three different vessels was conducted. 17 power spectra were measured for each ship.

The measurements were carried out using a multisensor mobile measuring platform designed and made by Polish Naval Academy and were brought off in the Gulf of Gdansk near the seaway to the port of Gdynia. The module was placed on the seabed at a depth of $20 \mathrm{~m}$ and connected with the ship-base by means of a hybrid electric-fiber optic cable of a length of $1 \mathrm{~km}$. In order to reduce the impact of noise from the ship-base, it was anchored at a distance of about $800 \mathrm{~m}$ from the measuring platform. Moreover, at the time when the measurements were performed, all sources of noise such as engines or generators were excluded, and all devices were

*corresponding author; e-mail: mwiciak@pk.edu.pl battery-powered. The recorded vessels sailed directly over the measuring module or close to it. The vessels' routes were recorded via AIS (Automatic Identification System) which contained information about the current position of the vessel [6].

Numerical experiments were performed using MATLAB. In each experiment, 16 samples of a vessel were randomly selected to form a training class. Then, for remaining 3 samples, one of each vessel (testing class), the Mahalanobis distance from each ship pattern was computed to find the closest one. The experimental results show that the presented method is effective, and results were close to those achieved in [3] even though the number of samples was small.

\section{Fractional Brownian motion}

Fractional Brownian motion ( $\mathrm{fBm}$ ) is recently widely used in engineering applications. The main reasons that make fBm encountered in applications are the selfsimilarity (which intuitively means that its behavior is stochastically the same, up to a space-scaling, regardless of the timescale), and short or long-memory of a process. And these two features are commonly observed in phenomena dealt with in physics, bioengineering, communications engineering, and finance [14-16].

By definition, the fractional Brownian motion with parameter $H(0<H<1)$ is a centered Gaussian process $B_{H}=\left\{B_{H}(t): 0 \leq t<\infty\right\}$ such that $B_{H}(0)=0$ and

$$
\boldsymbol{E}^{2}\left(B_{H}(t+s)-B_{H}(t)\right)=\boldsymbol{E}\left(B_{H}(1)\right) s^{2 H},
$$

where $\boldsymbol{E}$ denotes the expected value, and $t, s \geq 0$ represent time. The parameter $H$ is called the Hurst parameter [17]. From definition it is easy to see that $\mathrm{fBm}$ with parameter $H$ is $H$ self-similar process, i.e. $B_{H}(a t)$ has the same distribution as $a^{H} B_{H}(t)$ for any positive number $a$. It is known that the covariance function $\rho$ for $\mathrm{fBm}$ is given by the formula

$$
\rho(s, t)=\boldsymbol{E}\left(B_{H}(s) B_{H}(t)\right)=
$$




$$
\frac{1}{2}\left(s^{2 H}+t^{2 H}-(t-s)^{2 H}\right)
$$

for $0 \leq s<t$.

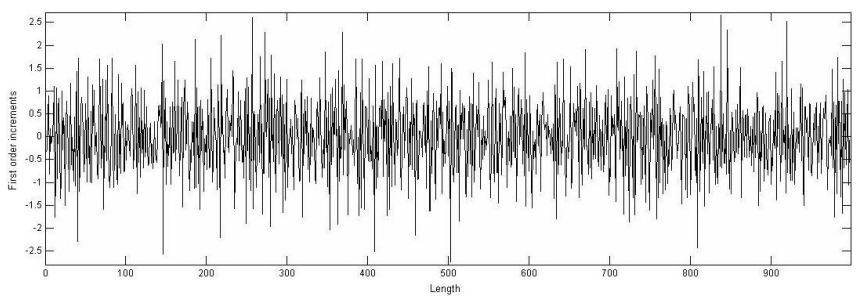

Fig. 1. First-order increments for fBm with $H=0.2$.

Let $X_{k}=B_{H}(k+1)-B_{H}(k)$ for $k=0,1, \ldots$ be the incremental process of $\mathrm{fBm} B_{H}$ (Fig. 1), and $\gamma_{k}=$ $E\left(X_{1} X_{k}\right)$ be the corresponding auto-covariance function. Then

$$
\gamma_{k}=\frac{1}{2}\left((k-1)^{2 H}-2 k^{2 H}+(k+1)^{2 H}\right)
$$

for $k=1, \ldots$, and the Hurst parameter $H$ determines the sign of covariance of future and past increments of $\mathrm{fBm}[15]$. In particular, if

- $H=\frac{1}{2}$, then $\operatorname{cov}\left(X_{n+1}, X_{n}\right)=0$, and according to property of Gaussian process $B_{H}$ has independent increments;

- $\frac{1}{2}<H<1$, then $\operatorname{cov}\left(X_{n+1}, X_{n}\right)>0$, and by the Taylor expansion for $f(x)=x^{2 H}$, one obtains $\gamma_{k} \approx H(2 H-1) k^{2 H-2}$, therefore the series $\sum_{k=1}^{\infty} \gamma_{k}=\infty$, and the $\mathrm{fBm}$ process $B_{H}$ is longrange dependent,

- $0<H<\frac{1}{2}$, then $\operatorname{cov}\left(X_{n+1}, X_{n}\right)<0$, and consequently the series $\sum_{k=1}^{\infty} \gamma_{k}<\infty$, and the $B_{H}$ is short-range dependent.

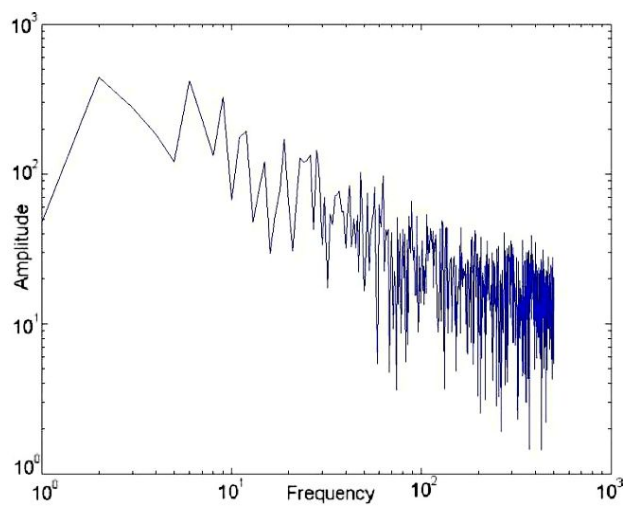

Fig. 2. Spectrum of fBm sample with $H=0.2$ in loglog plot.

Instead of analyzing stochastic processes in the time domain, processes can also be analyzed in the frequency or spectral domain [16]. A time-domain series can be transformed into a frequency-domain series, without any loss of information, by the Fourier transform. Figure 2 illustrates the spectrum of $\mathrm{fBm}$ with parameter $H=0.2$.

\section{Classification experiment}

The experiment was conducted for three different vessels. 17 power spectra were measured for each ship. In order to compensate different ships velocities, each sample was normalized by dividing by the maximum value in the spectrum. The power spectra of considered vessels are presented in Fig. 3. One can observe similarity of power spectra plots to the fBm one (Fig. 2).
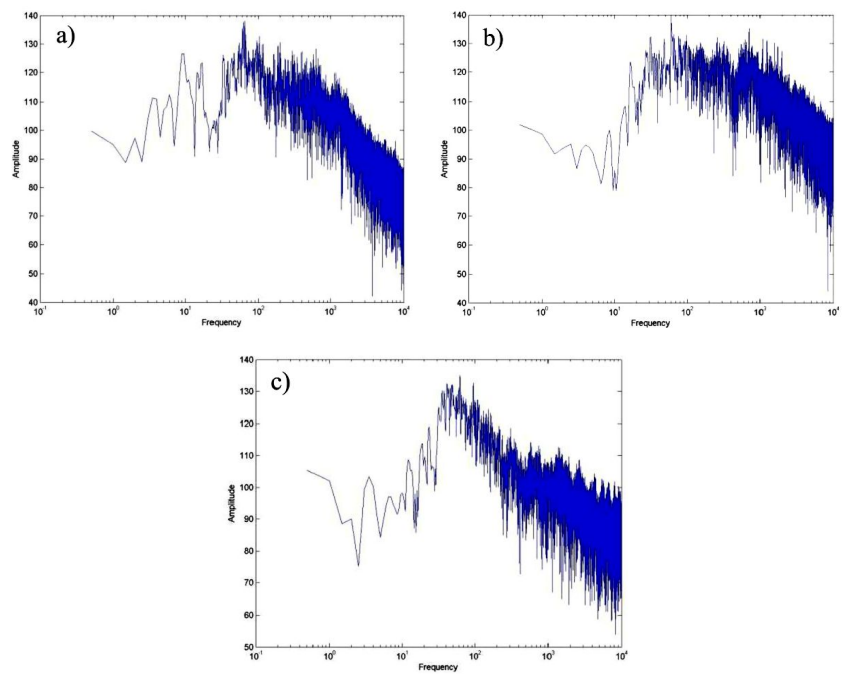

Fig. 3. Power spectra of three considered vessels: (a) ship A, (b) ship B, (c) ship C.

Let $\left(v_{1}, \ldots, v_{N}\right)$ denote values of the collected data. Define first-order increments sequence

$$
x_{0}=v_{1}, x_{n}=v_{n+1}-v_{n} \text { for } n=1, \ldots, N-1,
$$

and estimators for expectation and variance, respectively,

$$
\begin{aligned}
& \mu_{k}=\frac{1}{N-k} \sum_{i=1}^{N-k}\left(v_{i+k}-v_{i}\right) \text { for } k=1, \ldots, N-k, \\
& \sigma_{k}^{2}=\frac{1}{N-k} \sum_{i=1}^{N-k}\left(v_{i+k}-v_{i}-\mu_{k}\right)^{2} \\
& \quad \text { for } k=1, \ldots, N-k .
\end{aligned}
$$

For each sample, the sequences $\left.x_{n}: n=0,1, \ldots, N-1\right\}$ (first-order increments sequence, Fig. 4) and $\sigma_{k}: k=$ $1, \ldots, N-k$ (Fig. 5 ) were constructed. Obviously, by definition of $\mathrm{fBm}$, both sequences determine the Hurst parameter for ideal fractional Brownian motion. It should be admitted that ship sounds may not be ideal fBm signals and consequently, the increments of $v_{n}$ may change their distribution, fail to satisfy (1), and not give constant $H$; similarly, $\sigma_{k}$ may not be constant and differ for different $k$. However, it is assumed that sequences given by (4) and (6) provide information essential for 
the vessels identification. One can observe the similarity of first-order increments sequences for three vessels presented in Fig. 4 to that of fBm, Fig. 1.
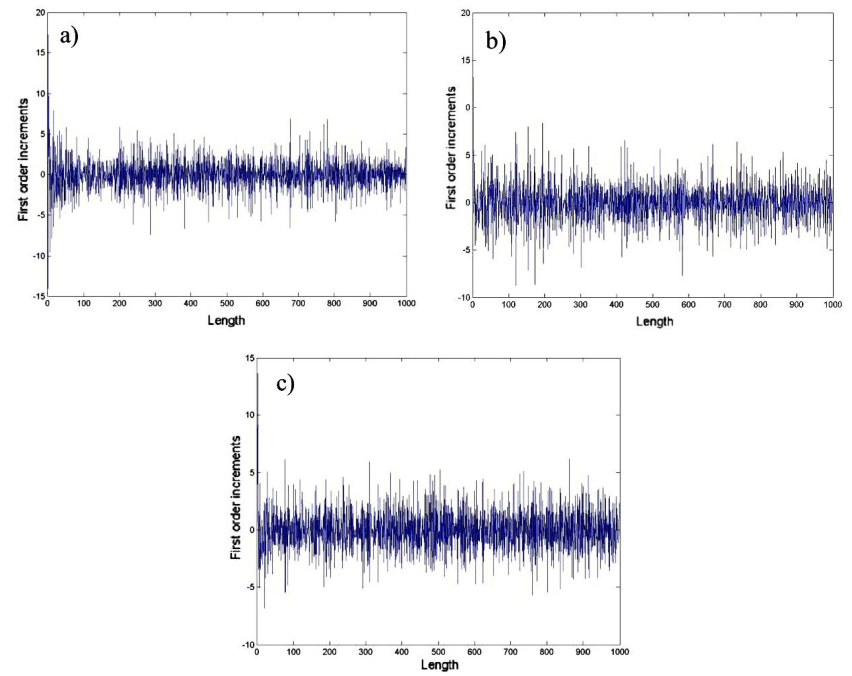

Fig. 4. First-order increments $x_{n}$, for three vessels: (a) ship A, (b) ship B, (c) ship C.
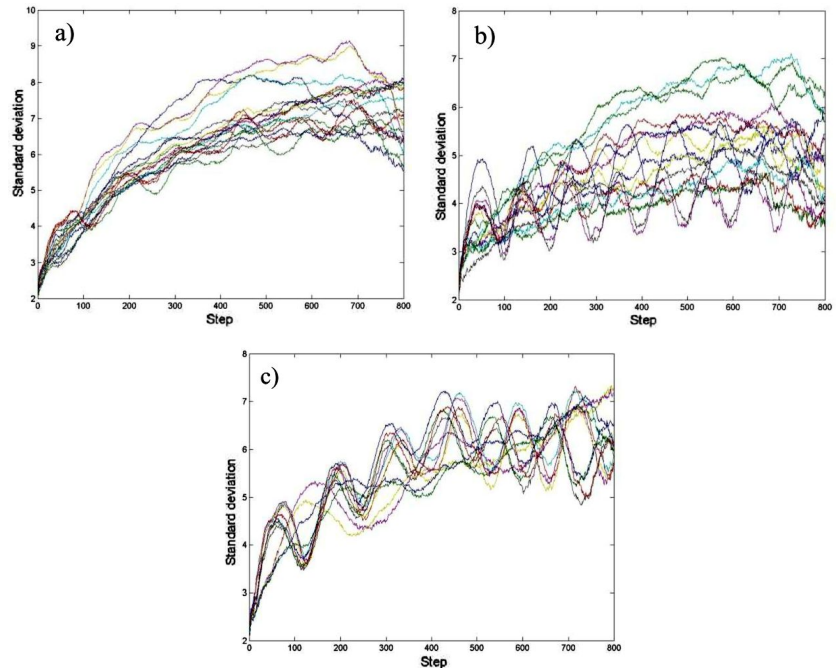

Fig. 5. $\left\{\sigma_{k}\right\}$ sequence for all 17 samples for three vessels: (a) ship A, (b) ship B, (c) ship C.

In the next step, 16 samples for each vessel were randomly selected to form a training class. Then, for remaining 3 samples, one of each vessel, forming the testing class, the Mahalanobis distance from each ship pattern was computed by the formula (for the squared distance)

$$
d^{2}(z, y)=(z-m) Q^{-1}(z-m)^{T},
$$

where $z=\left(x_{0}, x_{1}, \ldots, x_{t}\right)$ is testing observation $\left(x_{i}\right.$ for $i=0,1, \ldots, t$ given by formula (4)), $y$ is a group of observations for a given vessel i.e. $y=\left(y_{0}, y_{1}, \ldots, y_{t}\right)$, where $y_{i}=\left(x_{i}^{1}, \ldots, x_{i}^{16}\right)^{T}, i=0,1, \ldots, t$, and $t$ was assumed to be 10 due to small number of collected samples. $Q$ is the covariance matrix of random vector $y$,

$$
Q=\left[\operatorname{cov}\left(y_{i}, y_{j}\right)\right]_{i, j=0,1, \ldots, t}, \operatorname{cov}\left(y_{i}, y_{j}\right)=
$$

$$
\begin{gathered}
\frac{1}{16} \sum_{i=1}^{16}\left(x_{i}^{l}-m_{i}\right)\left(x_{j}^{l}-m_{j}\right), \\
m_{i}=\frac{1}{16} \sum_{l=1}^{16} x_{i}^{l}, m=\left(m_{0}, \ldots, m_{t}\right) .
\end{gathered}
$$

For each testing sample, three distances from three different patterns were computed, and the minimum values were found. Finally, decision was made to classify a testing sample as the one from a vessel with minimum Mahalanobis distance.

The similar computations were conducted using $\left\{\sigma_{k}\right\}$ sequence instead of $x_{n}$ as it was considered in [3]. The main difference was the small number of samples which enforced reduction of patterns.

All numerical experiments were repeated 3 times in series of 1000 drawings each, and were performed in MATLAB. The classification accuracy regarding three vessels and different classifier criteria is presented in Tables I and II.

\section{TABLE I}

Classification results using firstorder increments criterion.

\begin{tabular}{c|c|c}
\hline \hline $\begin{array}{c}\text { Vessel A } \\
{[\%]}\end{array}$ & $\begin{array}{c}\text { Vessel B } \\
{[\%]}\end{array}$ & $\begin{array}{c}\text { Vessel C } \\
{[\%]}\end{array}$ \\
\hline 94.1 & 100 & 57.6 \\
95.1 & 100 & 58.1 \\
94.3 & 100 & 61.4
\end{tabular}

TABLE II

Classification results using $\left\{\sigma_{k}\right\}$ sequence criterion.

\begin{tabular}{c|c|c}
\hline \hline $\begin{array}{c}\text { Vessel A } \\
{[\%]}\end{array}$ & $\begin{array}{c}\text { Vessel B } \\
{[\%]}\end{array}$ & $\begin{array}{c}\text { Vessel C } \\
{[\%]}\end{array}$ \\
\hline 92.5 & 94 & 67.7 \\
92.6 & 95.1 & 63.5 \\
93.8 & 93.3 & 68.3
\end{tabular}

The obtained results of the classification are adequate for vessel B. The method that used increment sequence gave $100 \%$ of correct answers, so the results are much better than those obtained when using $\left\{\sigma_{k}\right\}$ sequence criterion. For vessel A, identification results were still sufficient, slightly better when using $\left\{x_{n}\right\}$ sequence criterion, and similar to those obtained in [4] with the use of neural networks. For some reason, classification results were rather poor for vessel C. Present authors believe that a larger collection of samples would improve recognition results in the case of vessels $\mathrm{B}$ and $\mathrm{C}$.

\section{Conclusions}

The problem of vessel identification by its radiated sound was considered in this paper. It was shown that methods based on fractional Brownian analysis were effective in ship recognition. Two features were used to 
construct two different classifier criteria. The one based on increment sequence gave $100 \%$ of accuracy in recognizing ship B, even though the number of recorded samples was too small to obtain perfect accuracy with the use of the criterion based on standard deviation of increments sequence.

In the further research, a larger number of different vessels types, and most importantly a larger number of samples for each vessel, should be taken into consideration. Further, other features that characterize fBm like the Hurst parameter should be developed.

\section{Acknowledgments}

This study is a part of the research project OPOB 0067 03002 supported by The National Centre for Research and Development.

\section{References}

[1] G.B. Yang, L.G. Lu, D.Z. Gao, Y. Jiang, H.N. Liu, Arch. Acoust. 40, 11 (2015).

[2] A. Ozga, Arch. Acoust., 4, 645 (2014).

[3] S. Yang, Z. Li, X. Wang, J. Acoust. Soc. Am. 112, $172(2002)$.

[4] A. Żak, WSEAS Transactions on Signal Processing 4, 137 (2008).
[5] S.J. Malinowski, I. Gloza, Acta Acust. united Ac. 88, 718 (2002).

[6] I. Gloza, R. Józwiak, K. Buszman, Hydroacoustics 17, 63 (2014).

[7] E. Kozaczka, G. Grelowska, S. Kozaczka, W. Szymczak, Arch. Acoust. 38, 99 (2013).

[8] D. Zhao, Zh. Huand, Sh. Su, T. Li, Arch. Acoust, 38, 105 (2013).

[9] G. Grelowska, E. Kozaczka, S. Kozaczka, W. Szymczak, Arch. Acoust. 38, 351 (2013).

[10] G. Grelowska, E. Kozaczka, Arch. Acoust. 4, 439 (2014).

[11] J. Marszal, Arch. Acoust., 1, 103 (2014).

[12] R.J. Urick, Principles of Underwater Sound, 3rd ed, McGraw-Hill, New York 1983.

[13] M.S. Keshner, Proc. IEEE 70, 212 (1982).

[14] B. Mandelbrot, J. Van Ness, SIAM Rev. 10, 422 (1968).

[15] T. Sottinen, J Theor. Probab. 17, 309 (2004).

[16] A.B. Dieker, M. Mandjes, Informational Sciences Probability in the Engineering and Informational Sciences 17, 417 (2003).

[17] H.E. Hurst, R.P. Black, Y.M. Sinaika, Long Term Storage in Reservoirs. An Experimental Study, Constable, London, 1965. 